\title{
Does comorbid bipolar disorder increase neuropsychological impairment in children and adolescents with ADHD?
}

\author{
Joana C. Narvaez, ${ }^{1}$ Cristian P. Zeni, ${ }^{2}$ Roberta P. Coelho, ${ }^{2}$ Flavia Wagner, ${ }^{3}$ Gabriel F. Pheula, ${ }^{2}$ \\ Carla R. Ketzer, ${ }^{2}$ Clarissa M. Trentini, ${ }^{3}$ Silzá Tramontina, ${ }^{2}$ Luis A. Rohde ${ }^{2,4}$ \\ ${ }^{1}$ Graduate Program in Psychiatry, Universidade Federal do Rio Grande do Sul (UFRGS), Porto Alegre, RS, Brazil. ${ }^{2}$ Juvenile Bipolar Disorder \\ Outpatient Program (ProCAB), Division of Child and Adolescent Psychiatry, Hospital de Clínicas de Porto Alegre (HCPA), UFRGS, Porto \\ Alegre, RS, Brazil. ${ }^{3}$ Graduate Program in Psychology, UFRGS, Porto Alegre, RS, Brazil. ${ }^{4}$ National Institute of Developmental Psychiatry for \\ Children and Adolescents.
}

\begin{abstract}
Objective: To assess differences in executive functioning between children and adolescents with attention-deficit/hyperactivity disorder (ADHD) comorbid or not with bipolar disorder (BD), and to study the neuropsychological profile of subjects with the comorbidity in a clinical sample from a developing country.

Method: Case-control study comparing 23 participants with BD + ADHD and 85 ADHD-only subjects aged 6 to 17 years old. Both groups were drug-free. Executive function domains were assessed with the Stroop Test, the Wisconsin Card Sorting Test, and the Continuous Performance Test II.

Results: The group with juvenile BD + ADHD showed a significantly worse performance on the Stroop task, including time in color $(p=0.002)$, time in color-word $(p<0.001)$, interference, number or errors in color and color-word $(p=0.001)$, and number of errors in word cards $(p=0.028)$. No between-group differences were found in other tests.

Conclusions: Our findings suggest that ADHD-only and ADHD + BD do not show differences in inhibitory control and set-shifting domains. However, children and adolescents with BD and comorbid ADHD show greater impairment in processing speed and interference control. This suggests a potentially higher impairment in the dorsolateral prefrontal cortex and may be a potential neuropsychological signature of juvenile BD comorbid with ADHD.
\end{abstract}

Keywords: ADHD; bipolar disorder; children; neuropsychology

\section{Introduction}

Juvenile bipolar disorder (JBD) is a chronic condition characterized by mood swings in children and adolescents. This disorder causes significant family, social, and academic impairment. ${ }^{1,2} \mathrm{~A}$ recent meta-analysis found the prevalence of bipolar disorder (BD) in children and adolescents to be around $1.8 \%{ }^{3}$ Considering bipolar spectrum disorders, from 4 to $18 \%$ of children and adolescents referred to psychiatric evaluations satisfy the criteria for those disorders. ${ }^{4}$ Attention-deficit/hyperactivity disorder (ADHD) is present in about $5 \%$ of children and adolescents across different cultures. ${ }^{5}$ High rates of comorbidity between ADHD and BD are observed in childhood (over 60\%), especially in clinical samples. ${ }^{6}$ In a recent study, Donfrancesco et al. $^{7}$ demonstrated that the rate of children with a diagnosis of BD was higher in a sample of patients with ADHD, (29/

Correspondence: Luis Augusto Rohde, Serviço de Psiquiatria da Infância e Adolescência, Hospital de Clínicas de Porto Alegre, Rua Ramiro Barcelos, 2350, CEP 90035-903, Porto Alegre, RS, Brazil.

E-mail: Irohde@terra.com.br

Submitted Jan 03 2013, accepted Jul 072013.
$173,16.7 \%)$ compared with controls $(1 / 100,1 \%)(p<$ 0.001).

There has been a lot of debate on the frontiers between ADHD and JBD. Several studies tried to explore potential similarities and differences addressing epidemiological, etiological, phenomenological, and pathophysiological aspects. ${ }^{8}$ One promising area for further understanding specific pathophysiological characteristics and consequent differentiation between these disorders is neuropsychological assessment.

However, neuropsychological findings are not easily interpretable due to contradictory results. Comparisons have been performed between children and adolescents with $\mathrm{BD}$ and healthy controls in several neurocognitive domains, including executive functions, sustained attention, response flexibility, inhibition, working memory, and verbal learning. ${ }^{9-12}$ Comorbidity with ADHD had little or no significant effect on neurocognitive performance. Conversely, other studies, such as the one conducted by Pavuluri et al., ${ }^{13}$ found that patients with pediatric BD and ADHD had a worse performance in attentional and executive function (EF) domains than BD-only patients, despite illness state (manic or euthymic) or medication status. 
Few studies conducted a direct comparison between patients with ADHD alone, or comorbid with BD. Rucklidge et al. $^{14}$ compared healthy controls $(n=41)$, patients with $\operatorname{ADHD}(n=30), \operatorname{BD}(n=12)$, and $B D+$ ADHD ( $n=12)$. The authors found that ADHD-only and $A D H D+B D$ groups were more impaired when compared with healthy controls and BD-only groups, especially in processing and naming speed, working memory, and response inhibition. However, this investigation had a small sample size and part of the sample was on psychotropic medication during the assessment. Henin et al. ${ }^{15}$ compared the neuropsychological profile of patients with BD + ADHD $(n=73)$, ADHD alone $(n=102)$, and 120 healthy controls. No differences were detected between the ADHD + BD and ADHD alone groups, except in one measure of processing speed, where the comorbid group performed more poorly. Mattis et al. ${ }^{16}$ conducted a study of 15 euthymic patients with $B D, 20$ with $A D H D$, and 18 with $B D+$ $A D H D$ and found that those with $B D$ and $B D+A D H D$ had slower processing speed, and higher intraindividual variability.

In sum, the scarce literature available suggests that $A D H D$ and $B D$ might share or promote synergistic neuropsychological deficits when comorbid. Also, despite the evidence that cultural aspects might clearly interfere with the neuropsychological performance of individuals, we did not find any published investigation on neuropsychological differences between children with ADHD and $A D H D+B D$ outside the U.S. In fact, the literature on neurocognition in BD from different cultures is extremely deficient. ${ }^{17}$ Thus, our primary objective was to assess differences in executive functioning between children and adolescents with $A D H D$ comorbid or not with $B D$, and to study the neuropsychological profile of subjects with the comorbidity in a clinical sample from a developing country. Our primary hypothesis was that findings from previous studies in the U.S. documenting an additive effect of the comorbidity in measures of executive functioning in youths would be replicated in a different culture.

\section{Methods}

\section{Participants}

A case-control study was conducted. Twenty-three participants diagnosed with BD comorbid with ADHD were available from the baseline assessment (drug-free for at least 4 weeks) of a randomized double-blind, placebo-controlled trial of Aripiprazole ${ }^{18}$ (registered at www.clinicaltrials.gov under the identifier NCT00116259). This group was compared to 85 subjects with ADHD without comorbid mood disorders from the ADHD outpatient clinic at the Universidade Federal do Rio Grande do Sul (UFRGS), Brazil (Hospital de Clínicas de Porto Alegre, HCPA). The ADHD sample was also drug-free for at least 4 weeks. The final sample consisted of 108 participants aged from 6 to 17 years old, including both males and females.

\section{Diagnostic procedures and data collection}

Diagnostic process relied on the use of a semi-structured interview - KSADS-E ${ }^{19}$ - administered by trained investigators. All diagnoses generated were confirmed in clinical interviews using the DSM-IV criteria ${ }^{2}$ and conducted by experienced child and adolescent psychiatrists (see Rohde ${ }^{20}$ for a full description of the diagnostic assessment). ADHD onset prior to BD symptoms and a Young Mania Rating Scale (YMRS) ${ }^{21}$ score $>15$ were required at the baseline visit ${ }^{22}$ for the group with comorbid $\mathrm{BD}$ and ADHD. Exclusion criteria were use of psychotropic medication 4 weeks prior to entering the study; diagnoses of pervasive development disorder, schizophrenia, substance abuse or dependence, or mental retardation (estimated IQ: < 70); severe suicide/homicide risk counter-indicating outpatient treatment; any other acute or chronic disease; and pregnancy.

Participants verbally agreed to take part in the study, and parents provided written informed consent. This investigation was approved by the Ethics Committee of the HCPA.

\section{Instruments}

\section{Symptom assessment}

Mania symptoms were assessed with the YMRS. This is an 11-item scale for the assessment of severity of mania symptoms. ADHD symptoms were assessed using the Swanson, Nolan and Pelham Scale-Version IV (SNAPIV), a widely used scale in pharmacologic trials of $\mathrm{ADHD}^{23}$

\section{Neuropsychological assessment}

Executive function domains were assessed with the Stroop Test, ${ }^{24}$ the Wisconsin Card Sorting Test (WCST), ${ }^{25}$ and the Continuous Performance Test II (CPT II). ${ }^{26}$ Full scale IQ was estimated from Vocabulary and Block Design subtests of the Wechsler Intelligence Scale for Children-Third Edition. ${ }^{27}$ Tests were administered by experienced psychologists, following standard procedures and in the same order of administration for all participants, namely: Stroop Test, CPT II, and Wisconsin.

Stroop Test. This instrument was used to assess interference control, processing speed, and attention. Participants should read aloud three Stroop-cards: word card, the participant was asked to read a series of color words printed in a neutral color; color card, the individual was asked to name the color in which a bar of X's was printed; and color-word card, the individual was asked to name the color of the ink printed in conflicting color with the color words. Time and number of errors in each card were analyzed. In addition, interference score was calculated through the difference between time in colorword and color card.

CPT II. This is a measure of inhibitory control, including sustained attention, visual-motor speed, and impulsivity. Participants were instructed to press a button on the keyboard of the computer whenever any letter was shown on the screen, except the $\mathrm{X}$ letter. The inter-stimulus 
intervals (ISI) are 1, 2 and 4 seconds and the task takes 14 minutes. The analyzed scores were: omissions (missed targets), commissions (incorrect targets), hit response time (mean response time for all target responses), variability of standard error (SE), detectability (how well the individual discriminates between targets and nontargets), response style, perseverations (response that occurs less than 100 ms following a stimuli), hit RT block change (slope of change in reaction time [RT] over six time blocks) and hit RT ISI change (slope of change in RTs over the three ISIs, 1, 2 and 4 seconds).

WCST. This instrument measures the ability to solve abstract problems and set-shifting capacity. In the 128card computerized WCST, the subject was required to match a series of cards according to varying criteria. Subjects were not instructed about how cards should be matched, and they should apprehend the criterion according to the given feedback (correct or incorrect). The analyzed scores were total errors, perseverative responses, non-perseverative errors, perseverative errors, number of categories and trials to complete first category.

\section{Statistical analysis}

Descriptive and inferential multivariate analysis of variance (MANOVA) analyses were performed. Effect size was calculated using partial eta squared (according to Cohen $^{28}$ : eta squared $<0.06=$ small; $0.06-0.14=$ medium, $>0.14=$ large effect size). SPSS version 18.0 was used for all analyses. The level of significance accepted was 0.05 and all tests were two-tailed.

The potential covariates evaluated were age, sex, IQ, comorbidity (anxiety disorders, conduct disorder, and oppositional defiant disorder), and baseline score at the SNAP-IV. The former variables were chosen conceptually according to the literature. For the three neuropsychological tests - Wisconsin, CPT-II, and Stroop Test -, all potential confounders were included in the initial model. These possible confounding variables were statistically tested but were not significant, and therefore excluded from the final analysis by a backward elimination procedure. Logarithmic transformation was conducted to normalize the scores of all tests.

\section{Results}

Twenty-three children and adolescents with BD comorbid with ADHD, and eighty-five subjects with ADHD alone were available. Baseline demographic and clinical data are presented in Table 1. Of note, there were significant differences between BD + ADHD and ADHD-only groups in mean age $(p=0.03)$ and gender $(p=0.001)$, but not in mean IQ $(p=0.07)$. The $B D+A D H D$ group was older than the ADHD-only group, and most participants were girls in the group with the comorbidity.

We did not detect significant differences in WCST $\left(F_{6,99}=0.69, p=0.65\right.$, eta $\left.=0.040\right)$ scores between ADHD and ADHD + BD groups. Age and IQ were retained in the final model for the WCST analysis (Table 1). Also, between-group differences for the CPT II were not statistically significant $\left(F_{12,94}=0.86, p=0.59\right)$, but there was a medium effect size (eta $=0.099$ ). Although the effect sizes have all been small, individual score results show significant differences in commissions, hit RT SE, hit RT ISI, and hit RT ISI SE. The comorbid group showed a worse performance in all the reported measures. For the CPT II analysis, only age was retained as a covariate in the final model (Table 2).

The analysis conducted with the Stroop scores revealed significant group differences between ADHD and $A D H D+B D$ participants $\left(F_{7,98}=3.48, p=0.002\right.$, partial eta squared $=0.199)$ (Figure 1). All analyzed variables, including time, number of errors in all Stroop cards and Interference score were significantly different between groups, except for time in the word card task.

\begin{tabular}{|c|c|c|c|c|c|}
\hline \multirow[b]{2}{*}{ Characteristic } & \multicolumn{2}{|c|}{$B D+A D H D(n=23)$} & \multicolumn{2}{|c|}{ ADHD $(n=85)$} & \multirow[b]{2}{*}{$p$-value } \\
\hline & Mean $(n)$ & SD (\%) & Mean $(n)$ & $\mathrm{SD}(\%)$ & \\
\hline \multicolumn{6}{|l|}{ Demographic data } \\
\hline Age (years) & 12.17 & 2.69 & 10.79 & 2.66 & 0.03 \\
\hline Gender (male) & 10 & 43.5 & 66 & 77.6 & 0.001 \\
\hline \multicolumn{6}{|l|}{ Clinical data } \\
\hline BD type I & 18 & 78.3 & - & - & \\
\hline BD type II & 5 & 21.7 & & & \\
\hline ADHD combined type & 17 & 73.9 & 57 & 67.1 & 0.08 \\
\hline Disruptive behavioral disorders & 18 & 78.3 & 44 & 51.8 & 0.02 \\
\hline Anxiety disorders & 10 & 43.5 & 29 & 34.1 & 0.41 \\
\hline IQ & 100.34 & 13.5 & 93.95 & 15.18 & 0.07 \\
\hline \multicolumn{6}{|l|}{ Baseline measures } \\
\hline SNAP-IV & 2.04 & 0.52 & 1.46 & 0.49 & $<0.01$ \\
\hline YMRS & 36 & 8.05 & - & - & - \\
\hline CGI-S & 3.95 & 1.17 & 4.07 & 0.65 & 0.55 \\
\hline CGAS & 46.68 & 6.70 & 59.16 & 8.37 & $<0.01$ \\
\hline
\end{tabular}

$\mathrm{ADHD}$ = attention-deficit/hyperactivity disorder; BD = bipolar disorder; CGAS = Children's Global Assessment Scale; CGI-S = Clinical Global Impression-Severity Index; IQ = intelligence quotient; SD = standard deviation; SNAP-IV = Swanson, Nolan and Pelham Scale - Version IV; YMRS = Young Mania Rating Scale.

Comparison between groups using $t$ tests (continuous data) or Fisher's exact tests (categorical data). 
Table 2 ADHD-only and ADHD + BD performance in CPT II and WCST

\begin{tabular}{lccc}
\hline & $\begin{array}{c}\text { ADHD } \\
(\mathrm{n}=85)\end{array}$ & $\begin{array}{c}\text { ADHD + BD } \\
(\mathrm{n}=23)\end{array}$ & $\begin{array}{c}\text { ES (partial eta } \\
\text { squared) }\end{array}$ \\
\hline CPT II (t scores)* & & & 0.999 \\
Omissions & & & 0.59 \\
Commissions & $52.17(50.23-54.18)$ & $53.67(49.85-57.77)$ & 0.004 \\
Hit RT & $51.32(49.67-53.02)$ & $56.16(52.71-59.84)$ & 0.056 \\
Hit RT SE & $52.20(50.13-54.36)$ & $52.30(48.33-56.59)$ & 0.000 \\
Variability of SE & $52.17(50.15-54.27)$ & $57.31(53.08-61.89)$ & 0.042 \\
Detectability & $51.93(49.91-54.02)$ & $56.48(52.30-60.99)$ & 0.034 \\
Response style & $51.99(50.35-53.68)$ & $55.12(51.79-58.67)$ & 0.014 \\
Perseverations & $50.63(49.02-52.29)$ & $51.13(48.01-54.45)$ & 0.034 \\
Hit RT block change & $52.37(50.17-54.67)$ & $57.02(52.44-61.99)$ & 0.001 \\
Hit RT Block SE change & $49.12(47.23-51.08)$ & $51.38(47.61-55.45)$ & 0.029 \\
Hit RT ISI change & $48.41(46.44-50.46)$ & $50.61(46.67-54.87)$ & 0.010 \\
Hit RT ISI SE change & $48.31(46.13-50.59)$ & $53.93(49.29-58.99)$ & 0.009 \\
WCST (raw scores) & $48.89(46.92-50.94)$ & $54.02(49.87-58.52)$ & 0.703 \\
Total errors & & & 0.042 \\
Perseverative responses & & & 0.043 \\
Non-perseverative errors & $50.57(46.63-54.84)$ & $53.62(45.67-62.94)$ & 0.339 \\
Perseverative errors & $23.33(21.03-25.88)$ & $24.55(19.99-30.13)$ & 0.034 \\
Number of categories & $25.34(22.05-29.11)$ & $28.75(21.85-37.79)$ & 0.031 \\
Trials to complete first category & $21.65(19.74-23.74)$ & $22.24(18.53-26.69)$ & 0.004 \\
\hline
\end{tabular}

$\mathrm{ADHD}$ = attention-deficit/hyperactivity disorder; BD = bipolar disorder; CPT II = Continuous Performance Test II; ISI = inter-stimulus intervals; $\mathrm{RT}=$ reaction time; $\mathrm{SE}=$ standard error; WCST $=$ Wisconsin Card Sorting Test.

All results described as mean and $95 \%$ confidence interval.

* Age as covariate;

$\dagger$ age and IQ as covariates.

The ADHD + BD group performed significantly worse than the ADHD-only group. Age and IQ were retained as covariates in the final model.

\section{Discussion}

This study aimed to compare the neuropsychological profile of symptomatic children and adolescents presenting BD comorbid with ADHD with those suffering from ADHD without BD. We detected a significantly worse performance in the Stroop color-word test in the JBD + ADHD group, but no group differences in the WCST test and overall CPT II scores.

When considering CPT II individual scores, although there was no between-group difference in overall hit RT (average speed of correct responses for the entire test), the comorbid group had a significant worse performance in hit RT SE, which measures the response speed consistency. Additionally, hit RT ISI and hit RT ISI SE indicate change in mean RTs at the different ISIs (when
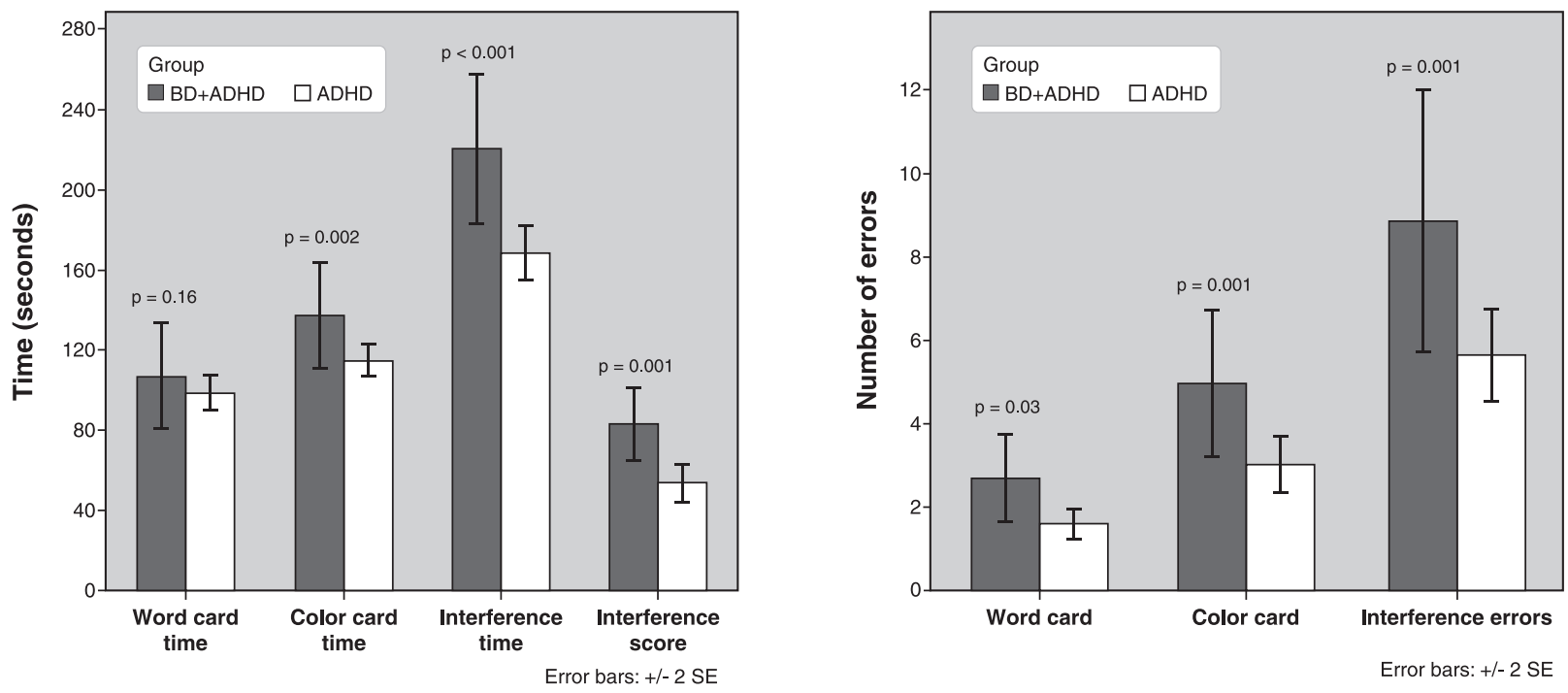

Figure 1 Stroop test performance in BD + ADHD and ADHD patients: differences in time and number of errors for each card and Interference score. Age and IQ were covariates; total difference between ADHD and ADHD + BD: $F_{7,98}=3.48, p=0.002$, partial eta squared $=0.199$. $A D H D=$ attention-deficit/hyperactivity disorder; $B D=$ bipolar disorder. 
stimulus are presented at 1,2, or 4-second intervals), suggesting that the comorbid group had slower and more variable RTs in response to longer ISIs. Mattis et al. ${ }^{16}$ suggest that slower and more variable RT as a function of longer ISIs may be a trait feature of pediatric BD. Otherwise, enlarged variability in RT related to slower event rates is already well documented for ADHD and is also present in other clinical conditions. ${ }^{29}$ Thus, longer and more variable RT related to larger event rates seem to be present in both disorders. ${ }^{30}$ As our results have shown, the comorbid group had a worse performance compared to ADHD-only.

Our findings in the Stroop Test indicate that processing speed, interference control, and accuracy are more impaired in the JBD + ADHD group. A study conducted by Doyle et al. ${ }^{12}$ compared children and adolescents with BD ( $n=57) ; 74 \%$ of these participants also had comorbid ADHD, and controls $(n=46)$. They found that JBD was associated with impairments on tasks reflecting sustaining attention, working memory, and processing speed (using the Stroop word and color cards) even after controlling for ADHD. The difference in the Stroop word card was not as robust as the difference in color card, possibly because reading is an overlearned and automatic process. Other studies corroborate these findings, even with the use of different processing speed tasks. ${ }^{16}$

We further analyzed the Stroop Interference score through the correction for baseline processing speed, thus constituting a more pure measure of interference control. ${ }^{12} \mathrm{~A}$ recent review reported that impairments in interference control are specific to $\mathrm{BD}{ }^{31}$ Our data suggests that $B D+A D H D$ patients show a decrement in interference control after correcting for baseline processing speed. This refinement was also conducted by Doyle et al., ${ }^{12}$ and differences between BD and control subjects (after statistic control for ADHD) emerged but were not significant, possibly due to the presence of higher rates of other comorbidities in their sample. In our sample, all comorbidities were statistically controlled according to our restrictive definition of confounding variables. Furthermore, interference control is a cognitive function that requires inhibiting capacity, sustained attention, and inhibitory break, including automatized and learning required processes, such as reading, and it was detected to be more impaired in the BD + ADHD group. Such results are in accordance with the clinical characteristics associated with JBD, such as behavioral lack of control, impulsivity, and aggressiveness. ${ }^{12}$

Abnormal brain structures and circuits have been suggested as additive substrates of the observed synergistic dysfunctional neurocognitive performance present in the comorbidity between JBD and ADHD. Left dorsolateral prefrontal cortex (DLPFC) and anterior cingulate cortex (ACC) have been associated with interference control assessed by the Stroop paradigm. ${ }^{32}$ It is well established that DLPFC and ACC are key impaired brain areas in ADHD. ${ }^{33}$ The most replicated alterations in ADHD in childhood include significantly smaller volumes in the dorsolateral prefrontal cortex, caudate, pallidum, corpus callosum, and cerebellum. ${ }^{34}$
DLPFC is also an important substrate of the dysfunctional neurocognitive performance in $\mathrm{JBD}^{35}$ and in the first manic episode of patients when inhibitory control tasks were assessed. ${ }^{36}$ Apparently, the impairment in brain circuits most replicated in both $A D H D^{34}$ and $\mathrm{BD}^{36}$ are related to the frontal and dorsolateral prefrontal cortex, being linked to inhibitory control tasks. Thus, impaired interference control might be a potential neuropsychological signature of JBD comorbid with ADHD.

Results from the CPT-II and WCST suggest that there are no differences in EF, specifically in the domains of response inhibition and sustained attention (assessed by the CPT II) and set-shifting (assessed by the WCST) between groups. A recent review by Walshaw et al. ${ }^{31}$ exploring specifically EF in patients with pediatric $B D$ and $A D H D$ found that response inhibition did not discriminate ADHD and BD groups, and the authors discussed that deficits in response inhibition were present in some degree in both groups. Findings in planning and setshifting, which were suggested to be specific to BD by Walshaw et al., ${ }^{31}$ were not observed in our data. However, planning was assessed using a different test (Tower of London), and the number of studies with the WCST was much smaller for BD than ADHD.

Our findings should be considered in the context of some limitations. There were not a BD-alone and neither typically developing groups in our study. However, it is important to note that, although many studies with larger samples have shown different neuropsychological profiles between healthy controls and specific mental disorders, investigations comparing children with comorbid diagnoses like ADHD and BD are scarce. ${ }^{12}$ Such studies may promote an advance in the neuropsychological understanding of the putative effect of both conditions. Moreover, the comorbidity between JBD and ADHD is the rule in clinical samples of children and adolescents. Therefore, it is a higher priority to understand differences between the comorbidity in terms of external validity. Although we cannot control our results for any fatigue effect, all instruments were administered to all subjects in the same order. We also did not include in our sample patients with BD in euthymia, thus manic symptoms might have interfered in the results. However, current studies suggest that neurocognitive impairments are trait-like characteristics of pediatric BD. ${ }^{37}$ Finally, we were not able to include instruments assessing other cognitive functions, such as verbal memory and working memory, consistently found to be impaired in BD. ${ }^{38}$

Overall our findings suggest that ADHD-only and ADHD + JBD patients do not show EF differences in the domains assessed by the CPT II and WCST. In contrast, children and adolescents presenting $\mathrm{BD}$ and comorbid ADHD show greater impairment in speed processing and interference control. As a result, previous studies conducted in the U.S. on the additive effect of the comorbidity in youths were replicated in a different culture, confirming our primary hypothesis. Therefore, our study provides evidence regarding the neuropsychological deficits of these patients, so that techniques to improve speed processing and interference control 
should be included in the psychotherapy for children and adolescents with BD + ADHD. Future studies should include a sample of BD-alone patients in order to identify specific and superposing cognitive deficits.

\section{Acknowledgements}

The authors would like to thank Natália Kapczinski and Anabel Martinez Arán for their thoughtful suggestions and comments in the manuscript and Aline Sokolovsky for helping to collect data. Cristian P. Zeni has received research grants from Conselho Nacional de Desenvolvimento Científico e Tecnológico (CNPq). Flavia Wagner has received research grants from Coordenação de Aperfeiçoamento de Pessoal de Nível Superior (CAPES). Clarissa M. Trentini has received research grants from CNPq and CAPES.

\section{Disclosure}

ST has received speaker's honoraria from Abbott Laboratories. LAR has been on the speakers' bureau/ advisory board and/or acted as consultant for Eli-Lilly, Janssen-Cilag, Novartis and Shire in the last three years. The ADHD and Juvenile Bipolar Disorder Outpatient Programs chaired by him received unrestricted educational and research support from the following pharmaceutical companies in the last three years: Eli-Lilly, Janssen-Cilag, Novartis, and Shire.

\section{References}

1 Zeni CP, Tramontina S, Ketzer CR, Pheula GF, Rohde LA Methylphenidate combined with aripiprazole in children and adolescents with bipolar disorder and attention-deficit/hyperactivity disorder: a randomized crossover trial. J Child Adolesc Psychopharmacol. 2009;19:553-61.

2 American Psychiatric Association. Diagnostic and statistical manual of mental disorders - DSM-IV-TR ${ }^{\circledR}$. Washington: American Psychiatric Publishing; 2000.

3 Van Meter AR, Moreira AL, Youngstrom EA. Meta-analysis of epidemiologic studies of pediatric bipolar disorder. J Clin Psychiatry. 2011;72:1250-6.

4 Soutullo CA, Chang KD, Díez-Suárez A, Figueroa-Quintana A, Escamilla-Canales I, Rapado-Castro M, et al. Bipolar disorder in children and adolescents: international perspective on epidemiology and phenomenology. Bipolar Disord. 2005;7:497-506.

5 Polanczyk G, de Lima MS, Horta BL, Biederman J, Rohde LA. The worldwide prevalence of ADHD: a systematic review and metaregression analysis. Am J Psychiatry. 2007;164:942-8.

6 Kowatch RA, Youngstrom EA, Danielyan A, Findling RL. Review and meta-analysis of the phenomenology and clinical characteristics of mania in children and adolescents. Bipolar Disord. 2005;7:483-96.

7 Donfrancesco R, Miano S, Martines F, Ferrante L, Melegari MG, Masi G. Bipolar disorder co-morbidity in children with attention deficit hyperactivity disorder. Psychiatry Res. 2011;186:333-7.

8 Galanter CA, Leibenluft E. Frontiers between attention deficit hyperactivity disorder and bipolar disorder. Child Adolesc Psychiatr Clin N Am. 2008;17:325-46, viii-ix.

9 DelBello MP, Adler CM, Amicone J, Mills NP, Shear PK, Warner J, et al. Parametric neurocognitive task design: a pilot study of sustained attention in adolescents with bipolar disorder. J Affect Disord. 2004;82:S79-88.

10 McClure EB, Treland JE, Snow J, Dickstein DP, Towbin KE, Charney $\mathrm{DS}$, et al. Memory and learning in pediatric bipolar disorder. J Am Acad Child Adolesc Psychiatry. 2005;44(5):461-9.
11 Dickstein DP, Treland JE, Snow J, McClure EB, Mehta MS, Towbin $\mathrm{KE}$, et al. Neuropsychological performance in pediatric bipolar disorder. Biol Psychiatry. 2004;55:32-9.

12 Doyle AE, Wilens TE, Kwon A, Seidman LJ, Faraone SV, Fried R, et al. Neuropsychological functioning in youth with bipolar disorder. Biol Psychiatry. 2005;58:540-8.

13 Pavuluri MN, Schenkel LS, Aryal S, Harral EM, Hill SK, Herbener ES, et al. Neurocognitive function in unmedicated manic and medicated euthymic pediatric bipolar patients. Am J Psychiatry. 2006;163:28693.

14 Rucklidge JJ. Impact of ADHD on the neurocognitive functioning of adolescents with bipolar disorder. Biol Psychiatry. 2006;60:921-8.

15 Henin A, Mick E, Biederman J, Fried R, Wozniak J, Faraone SV, et al. Can bipolar disorder-specific neuropsychological impairments in children be identified? J Consult Clin Psychol. 2007;75:210-20.

16 Mattis S, Papolos D, Luck D, Cockerham M, Thode HC Jr. Neuropsychological factors differentiating treated children with pediatric bipolar disorder from those with attention-deficit/hyperactivity disorder. J Clin Exp Neuropsychol. 2011;33:74-84.

17 Tramontina S, Zeni CP, Rohde LA. Pediatric Bipolar Disorder: from the perspective of Brazil and South America. In: Diler RS, editor. Pediatric bipolar disorder: a global perspective. New York: Nova Science Publishers; 2007. p. 57-69.

18 Tramontina S, Zeni CP, Ketzer CR, Pheula GF, Narvaez J, Rohde LA. Aripiprazole in children and adolescents with bipolar disorder comorbid with attention-deficit/hyperactivity disorder: a pilot randomized clinical trial. J Clin Psychiatry. 2009;70:756-64.

19 Orvaschel $\mathrm{H}$. Psychiatric interviews suitable for use in research with children and adolescents. Psychopharmacol Bull. 1985;21:737-45.

20 Rohde LA. ADHD in Brazil: the DSM-IV criteria in a culturally different population. J Am Acad Child Adolesc Psychiatry. 2002;41:1131-3.

21 Young RC, Biggs JT, Ziegler VE, Meyer DA. A rating scale for mania: reliability, validity and sensitivity. Br J Psychiatry. 1978;133:429-35.

22 Biederman J, Mick E, Hammerness $P$, Harpold T, Aleardi M, Dougherty $M$, et al. Open-label, 8-week trial of olanzapine and risperidone for the treatment of bipolar disorder in preschool-age children. Biol Psychiatry. 2005;58:589-94.

23 A 14-month randomized clinical trial of treatment strategies for attention-deficit/hyperactivity disorder. The MTA Cooperative Group. Multimodal Treatment Study of Children with ADHD. Arch Gen Psychiatry. 1999;56:1073-86.

24 Comalli PE Jr, Wapner S, Werner H. Interference effects of Stroop color-word test in childhood, adulthood, and aging. J Genet Psychol. 1962;100:47-53.

25 Heaton RK, Chelune GJ, Talley JL, Kay GG, Curtiss G. Wisconsin Card Sorting Test manual (revised and expanded). Odessa: Psychological Assessment Resources; 1993.

26 Conners C. Conners' Continuous Performance Test (CPT II). North Tonawanda: Multi-Health Systems; 2000.

27 Wechsler D. Wechsler Intelligence Scale for children. 3rd ed. San Antonio: The Psychological Corporation; 1991.

28 Cohen J. Statistical power analysis for the behavioral sciences: L. Erlbaum Associates; 1988.

29 Tamm L, Narad ME, Antonini TN, O'Brien KM, Hawk LW Jr, Epstein JN. Reaction time variability in ADHD: a review. Neurotherapeutics. 2012;9:500-8.

30 Skirrow C, Hosang GM, Farmer AE, Asherson P. An update on the debated association between ADHD and bipolar disorder across the lifespan. J Affect Disord. 2012;141:143-59.

31 Walshaw PD, Alloy LB, Sabb FW. Executive function in pediatric bipolar disorder and attention-deficit hyperactivity disorder: in search of distinct phenotypic profiles. Neuropsychol Rev. 2010;20:103-20.

32 Nee DE, Wager TD, Jonides J. Interference resolution: insights from a meta-analysis of neuroimaging tasks. Cogn Affect Behav Neurosci. 2007;7:1-17.

33 Dickstein SG, Bannon K, Castellanos FX, Milham MP. The neural correlates of attention deficit hyperactivity disorder: an ALE metaanalysis. J Child Psychol Psychiatry. 2006;47:1051-62.

34 Seidman LJ, Valera EM, Makris N. Structural brain imaging of attention-deficit/hyperactivity disorder. Biol Psychiatry. 2005;57: 1263-72.

35 Pavuluri MN, Bogarapu S. Brain model for pediatric bipolar disorder. In: Banaschewski T, Rohde LA, editors. Biological child psychiatry: 
recent trends and developments. Advances in biological psychiatry. 24. Basel: Karger; 2008. p. 39-52.

36 Janssen J, Reig S, Parellada M, Moreno D, Graell M, Fraguas D, et al. Regional gray matter volume deficits in adolescents with firstepisode psychosis. J Am Acad Child Adolesc Psychiatry. 2008;47: $1311-20$
37 Pavuluri MN, Schenkel LS, Aryal S, Harral EM, Hill SK, Herbener ES, et al. Neurocognitive function in unmedicated manic and medicated euthymic pediatric bipolar patients. Am J Psychiatry. 2006;163:286-93.

38 Horn K, Roessner V, Holtmann M. Neurocognitive performance in children and adolescents with bipolar disorder: a review. Eur Child Adolesc Psychiatry. 2011;20:433-50. 